\title{
“Super” Cocktails for Heavy Ion Testing
}

\author{
Michael B. Johnson, Margaret A. McMahan, Michelle Galloway, Daniela Leitner, James R. Morel, \\ Thomas L. Gimpel, Brien F. Ninemire, Reba Siero, Raymond K. Thatcher
}

\begin{abstract}
The 4.5 MeV/nucleon heavy ion cocktail at the 88-Inch Cyclotron has been expanded by incorporating beams from solid material to fill in the linear energy transfer curve. This supercocktail is available by special request and is useful when only normal incidence between the beam and the device under test is possible or desirable.
\end{abstract}

Index Terms-BASE Facility, Cyclotron, Radiation Effects Testing, Heavy Ions, Cocktail, Supercocktail.

\section{INTRODUCTION}

$\mathrm{T}$ HE 88-Inch Cyclotron, part of the Nuclear Science Division at Lawrence Berkeley National Laboratory, is the home of the Berkeley Accelerator Space Effects (BASE) Facility and supports a local research program in nuclear science. A facility of the Department of Energy's (DOE) Office of Science, operation of the Cyclotron is jointly funded by the DOE, the United States Air Force, and the National Reconnaissance Office.

The BASE Facility [1] provides well-characterized beams of heavy ions, protons, and other medium energy particles that simulate the space environment. The largest part of the BASE program is the heavy ion testing, which utilizes the combination of Electron Cyclotron Resonance (ECR) ion sources and the 88-Inch Cyclotron to create "cocktail" beams [2]. A cocktail consists of a set of ions with nearly identical mass-to-charge $(\mathrm{A} / \mathrm{q})$ ratio that are tuned out together from the ion source and injected into the cyclotron. The cyclotron acts as a mass analyzer to separate out the individual ions of the cocktail. This technique allows for rapid ion changes over a large linear energy transfer (LET) range by simply adjusting cyclotron acceleration frequency and making minor adjustments to ion source controls.

In recent years, the testing community has increasingly required testing with a larger variety of ions, primarily due to the need to test only at normal incidence to the device under test (DUT). This increased demand is due in part to DUTs that have to be tested in a liquid-nitrogen dewar and thus cannot be rotated, and in part to DUTs that show geometric effects when tested at larger angles.

This work was supported by the U.S. Department of Energy under contract No. DE-AC02-05CH11231.

All authors are with Lawrence Berkeley National Laboratory's 88-Inch Cyclotron, Berkeley, CA 94720 USA (phone: 510-486-4389; fax: 510-4866122; e-mail: mbjohnson@lbl.gov).
The 88-Inch Cyclotron Operations staff have met these demands by developing a "supercocktail' version of the standard 4.5A MeV cocktail beam. The additional components of the cocktail have been incorporated through the addition of a multi-port sputter probe to the Advanced ECR ion source, the development of methods for adjusting the cyclotron frequency over a wider range, and utilization of two ion sources, the LBNL-ECR and the Advanced ECR (AECR-U).

\section{METHODS AND CAPABILITIES}

\section{A. The Supercocktail}

As ECR ion source technology advanced over the years, cocktail beams at four energies $(4.5,10,16$ and $32.5 \mathrm{~A} \mathrm{MeV})$ were developed for single event testing. The lower energy cocktail beams provide the widest span of LET values, while the higher energy cocktail beams are useful when higher range ions are needed. The standard 4.5A MeV cocktail consists of a mixture of gases $(\mathrm{N}, \mathrm{Ne}, \mathrm{Ar}, \mathrm{Kr}, \mathrm{Xe})$ and metallic ions $(\mathrm{B}, \mathrm{Cu}$, and $\mathrm{Bi}$ ). These ions cover the range of LETs from $1.7-99.7$ $\mathrm{MeV} /\left(\mathrm{mg} / \mathrm{cm}^{2}\right)$.

In a typical single event effect test, the LETs available from the standard cocktail beams are augmented by rotating the DUTs to increase the effective thickness of the material. A rotation as large as $60^{\circ}$ will produce an effective LET greater by a factor of two, resulting in a maximum LET - using $\mathrm{Bi}$ - of $200 \mathrm{MeV} /\left(\mathrm{mg} / \mathrm{cm}^{2}\right)$. Filling in the LET curve in this manner is sufficient for many applications.

In order to satisfy the applications that cannot use rotation to fill in the LET curve, we have combined several methods to produce beams from solid material - high and low temperature ovens, direct insertion, sputtering, and the 'metal ions from volatile compounds' (MIVOC) method - to add additional ions to the standard cocktail beam at $4.5 \mathrm{~A} \mathrm{MeV}$ to make a supercocktail. An LET curve for the supercocktail is provided in Fig. 1, and Table I lists the ions and LETs [3] for normal and $60^{\circ}$ incidence. Also shown in Fig. 2 are the full Bragg curves for all fourteen supercocktail ions.

The supercocktail has been made possible by the development of a radial sputter probe [4] for the AECR-U. Negative voltage is applied to the probe to incite collisions of plasma ions with the target atoms, thereby sputtering material into the ion source plasma. The new sputter probe has three heads and we are currently developing the electronics to bias three separate probes independently. 


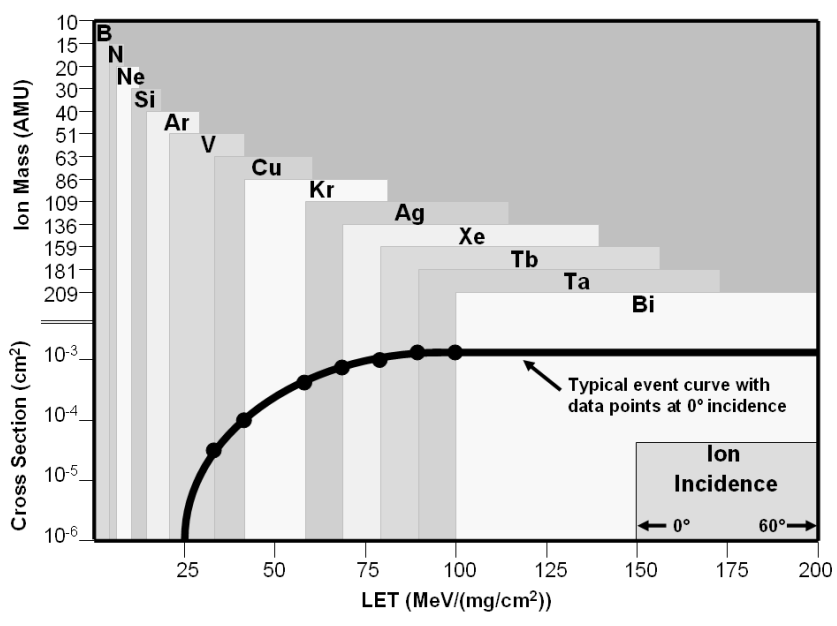

Fig. 1. Cross Section vs. LET Curve. The lateral width of each ion indicates the LET range that can be reached through rotation.

\section{B. Effect on Operations}

The 88-Inch Cyclotron serves dual missions, and scheduling constraints often dictate that one or more ovens be dedicated to nuclear science needs at any given time. Requests for the supercocktail need to be made when beamtime is requested, or all ions may not be available.

The number of ions requested in the supercocktail add to the time required for initial beam tune up and calibration, especially if both the AECR-U and LBNL-ECR ions sources are utilized, or if there are additional constraints like high-flux density for the high-LET ions. Flux density is monitored using four YAP:Ce scintillator detectors that are calibrated to a center detector for each ion at the beginning of the run. This calibration procedure also requires more time for the supercocktail.

TABLE I. Components of the 4.5 MeV/nucleon Supercocktail
Once the initial tuneup is completed, approximate times to switch between ions in the cocktail beam are given in Table II. Additional time is required to switch between the 'low-LET' and 'high-LET' portions of the cocktail, if both sources are utilized. Operation of the supercocktail requires a delicate balance of the ion source parameters. Good communication between the users and the Control Room throughout the course of the run is of prime importance.

\section{Quality Assurance}

The cocktail method has some fundamental limitations. Firstly, the A/q of the ion species cannot be too far from the pilot beam used to tune the cocktail, otherwise the injection lines and beamlines would have to be retuned. At the cyclotron, a maximum frequency range of approximately $\pm 400 \mathrm{kHz}$ (out of $14.3 \mathrm{MHz}$ for $4.5 \mathrm{MeV} /$ nucleon) provides a well-resolved cocktail tune. This translates to an A/q of $5.0 \pm 0.17$; all of the ions listed in Table I fall into this range. Secondly, the method is limited by the mass resolution of the cyclotron, which determines its ability to cleanly separate out ions with very close values of $\mathrm{A} / \mathrm{q}$. The frequency resolution - proportional to the mass resolution - of the 88-Inch Cyclotron is approximately $3 \mathrm{kHz}$. If two ions are similar enough in $\mathrm{A} / \mathrm{q}$ to fall within this resolution - or if a tail of a very intense beam extends into the frequency region of a weaker beam - the beam will be contaminated and may be unusable. The history of the ion source must also be considered, as some species can persist in the ion source for months after they have been run.

To ensure that only the desired ion is present in the beam, several techniques are employed. Taking advantage of the high efficiency of ECR sources, enriched isotopes are used to provide maximum flux density while avoiding beam contamination. Additionally, our operators have identified new methodologies that enable higher frequencies of operation, allowing further separation between desired and contaminant

\begin{tabular}{|c|c|c|c|c|c|c|c|c|c|}
\hline Ion & $\begin{array}{c}\text { Energy } \\
(\mathrm{MeV})\end{array}$ & $\bar{Z}$ & $\bar{A}$ & $\begin{array}{l}\text { Chg. } \\
\text { State }\end{array}$ & $\begin{array}{l}\% \text { Nat. } \\
\text { Abund. }\end{array}$ & $\begin{array}{l}\text { LET O } \\
(\mathrm{M} \mathrm{eV} /(\mathrm{m}\end{array}$ & $\begin{array}{l}\text { LET } 60^{\circ} \\
\left.\mathrm{g} / \mathrm{cm}^{2}\right) \text { ) }\end{array}$ & $\begin{array}{c}\text { Range } \\
(\mu \mathrm{m})\end{array}$ & Method \\
\hline$\overline{\mathbf{B}}$ & 44.9 & 5 & 10 & +2 & 19.9 & 1.65 & 3.30 & 78.5 & MIVOC \\
\hline $\mathbf{N}$ & 67.4 & 7 & 15 & +3 & 0.37 & 3.08 & 6.16 & 67.8 & Gas \\
\hline $\mathrm{Ne}$ & 90.0 & 10 & 20 & +4 & 90.48 & 5.77 & 11.54 & 53.1 & Gas \\
\hline $\mathbf{S} \mathbf{i}^{1,2}$ & 139.6 & 14 & 29 & +6 & 4.67 & 9.28 & 18.56 & 52.4 & Gas \\
\hline$A r$ & 180.0 & 18 & 40 & +8 & 99.6 & 14.32 & 28.64 & 48.3 & Gas \\
\hline $\mathbf{V}^{1,2}$ & 221.0 & 23 & 51 & +10 & 99.75 & 21.68 & 43.36 & 42.5 & Oven \\
\hline $\mathrm{Cu}$ & 301.8 & 29 & 63 & +13 & 69.17 & 29.33 & 58.66 & 45.6 & Probe \\
\hline $\mathrm{Kr}$ & 378.1 & 36 & 86 & +17 & 17.3 & 39.25 & 78.50 & 47.1 & Gas \\
\hline $\mathbf{Y}^{1,2}$ & 409.6 & 39 & 89 & +18 & 100 & 45.58 & 91.16 & 45.8 & Oven \\
\hline $\mathbf{A} \mathbf{g}^{1,2}$ & 499.5 & 47 & 109 & +22 & 48.161 & 58.18 & 116.36 & 46.3 & Oven \\
\hline $\mathrm{Xe}$ & 602.9 & 54 & 136 & +27 & 8.9 & 68.84 & 137.68 & 48.3 & Gas \\
\hline $\mathbf{T} \mathbf{b}^{1}$ & 724.2 & 65 & 159 & +32 & 100 & 77.52 & 155.04 & 52.4 & Probe \\
\hline $\mathbf{T} \mathbf{a}^{1}$ & 805.0 & 73 & 181 & +36 & 99.988 & 87.15 & 174.30 & 53.0 & Probe \\
\hline B i & 904.2 & 83 & 209 & +41 & 100 & 99.74 & 199.48 & 52.9 & Oven \\
\hline
\end{tabular}

${ }^{1}$ New supercocktail ions ${ }^{2} \mathrm{By}$ special request 


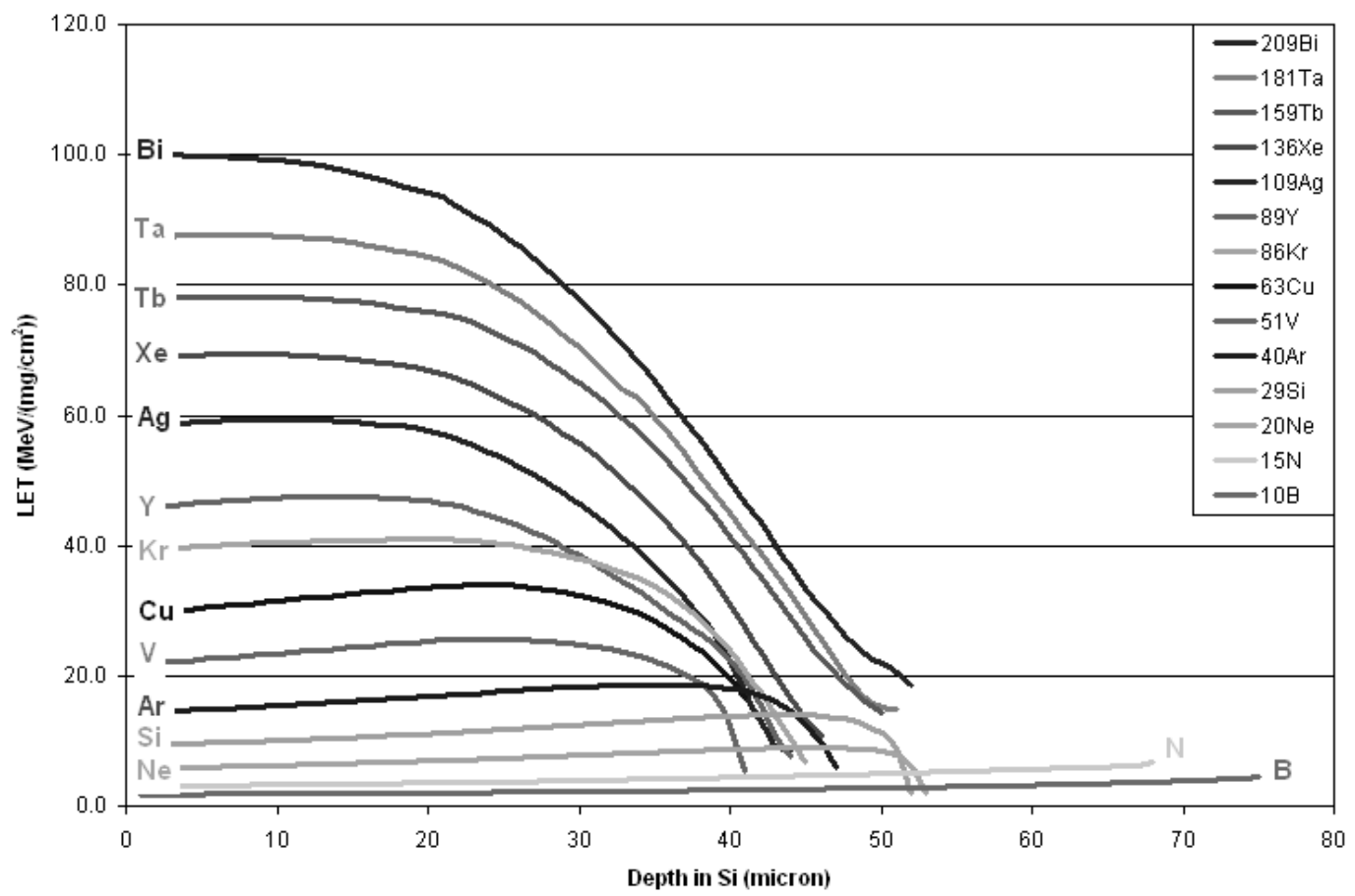

Fig. 2. 4.5A MeV supercocktail Bragg curves. These curves allow the user to correlate energy deposition with depth in silicon.

ions. Lastly, a silicon surface barrier detector is used. The detector signal is processed through a multi-channel analyzer (MCA) to confirm that the beam is pure. This system has been recently upgraded with thicker silicon barrier detector and a new preamplifier to ensure optimum performance for all energies of cocktail beams.

\section{Higher Energy Cocktail beams}

The supercocktail beam has been developed for the $4.5 \mathrm{~A}$ $\mathrm{MeV}$ cocktail specifically. However, some of the lower LET components, namely V, Y and $\mathrm{Ag}$, are also available in the 10A $\mathrm{MeV}$ cocktail, again by special request at the time beam is scheduled. The LET values associated with these components are available upon request.

\section{FUTURE UPGRADES}

A third ion source, VENUS, was coupled to the 88-Inch Cyclotron in 2006 and is undergoing commissioning experiments at the present time. VENUS is a third-generation, superconducting ion source that will enable higher intensities of heavy, high-charge state ions [5]. The source will be available for use by the BASE community once it is outfitted with gas ports, ovens, and sputter probes. A new set of attenuators has been installed between VENUS and the 88-Inch Cyclotron to enable adjustment of the relative intensity of cocktail components. VENUS will enable us to further expand the capability of the cocktail beams.
TABLE II. Time Required to Change Ions (in minutes)

\section{Finishing lon}

\begin{tabular}{|c|c|c|c|c|c|c|c|c|c|c|c|c|c|}
\hline & B & $\mathbf{N}$ & $\mathrm{Ne}$ & & $\mathrm{Ar}$ & $\mathbf{V}$ & $\mathrm{Cu}$ & $\mathrm{Kr}$ & $\mathbf{A g}$ & $X e$ & $\mathrm{~Tb}$ & $\mathrm{Ta}$ & \\
\hline B & - & 2 & 2 & 5 & 2 & 2 & 5 & $2^{*}$ & $2^{*}$ & $2^{*}$ & $2^{*}$ & $2^{*}$ & $2^{\star}$ \\
\hline $\mathbf{N}$ & 2 & - & 2 & 5 & 2 & 2 & 5 & $2^{*}$ & $2^{*}$ & $2^{*}$ & $2^{*}$ & $2^{*}$ & $2^{*}$ \\
\hline $\mathrm{Ne}$ & 2 & 2 & - & 5 & 2 & 2 & 5 & $2^{*}$ & $2^{*}$ & $2^{*}$ & $2^{*}$ & $2^{*}$ & $2^{*}$ \\
\hline $\mathrm{S}$ & 5 & 5 & 5 & - & 5 & 5 & 2 & $5^{\star}$ & $5^{\star}$ & $5^{*}$ & $5^{\star}$ & $5^{\star}$ & $5^{\star}$ \\
\hline Ar & 2 & 2 & 2 & 5 & - & 2 & 5 & $2^{*}$ & $2^{*}$ & $2^{*}$ & $2^{*}$ & $2^{*}$ & $2^{*}$ \\
\hline V & 2 & 2 & 2 & 5 & 2 & - & 5 & $2^{*}$ & $2^{*}$ & $2^{*}$ & $2^{*}$ & $2^{*}$ & $2^{*}$ \\
\hline $\mathrm{Cu}$ & 5 & 5 & 5 & 2 & 5 & 5 & & $5^{\star}$ & $5^{*}$ & $5^{*}$ & $5^{*}$ & $5^{\star}$ & $5^{\star}$ \\
\hline K & $2^{*}$ & $2^{*}$ & $2^{*}$ & $5^{\star}$ & $2^{*}$ & $2^{*}$ & $5^{*}$ & & 2 & 2 & 2 & 2 & 2 \\
\hline A & $2^{*}$ & $2^{*}$ & $2^{*}$ & $5^{\star}$ & $2^{*}$ & $2^{*}$ & $5^{\star}$ & 2 & - & 2 & 2 & 2 & 2 \\
\hline $\mathbf{X}$ & $2^{*}$ & $2^{*}$ & $2^{*}$ & $5^{\star}$ & $2^{*}$ & $2^{*}$ & $5^{\star}$ & 2 & 2 & - & 2 & 2 & 2 \\
\hline 1 & $2^{*}$ & $2^{\star}$ & $2^{*}$ & $5^{\star}$ & $2^{*}$ & $2^{*}$ & $5^{\star}$ & 2 & 2 & 2 & - & 2 & 2 \\
\hline $\mathbf{T}$ & $2^{*}$ & $2^{\star}$ & $2^{*}$ & $5^{\star}$ & $2^{*}$ & $2^{*}$ & $5^{\star}$ & 2 & 2 & 2 & 2 & - & 2 \\
\hline B & $2^{*}$ & $2^{*}$ & $2^{*}$ & $5^{\star}$ & $2^{*}$ & $2^{*}$ & $5^{\star}$ & 2 & 2 & 2 & 2 & 2 & - \\
\hline
\end{tabular}

\section{CONCLUSIONS}

The supercocktail adds new capabilities to the BASE Facility, and has been developed in response to the needs of our users. The ability to test devices with a wide selection of ions at $0^{\circ}$ incidence can be very useful when there are cooling or geometric constraints. With rapid ion switching, testing is cost-effective for our customers. 


\section{REFERENCES}

[1] M.A. McMahan, et al., Nucl Instr Meth. B241, 409 (2005)

[2] M.A. McMahan, et. Al., Nucl. Instr. Meth. A253, 1 (1986)

[3] J.F. Zeigler, SRIM-2006, http://www.srim.org/

[4] M. Galloway, et. al., High Energy Physics And Nuclear Physics 2007, Vol.31(S1): 75-79

[5] D. Leitner, et. al., Nucl. Instr. Meth. B235 486 (2005) 